\title{
La investigación en las instituciones de educación superior: Vinculación con el sector gobierno e industria (1981-2011)
}

\author{
Research in higher education institutions: Link with the government and industry \\ sector (1981-2011)
}

\author{
RODRÍGUEZ-MIRAMONTES, Jorge †* \& HERNÁNDEZ-GARCÍA, Maritza
}

Universidad Politécnica del Valle de México; Av. Mexiquense s/n esquina Av. Universidad Politécnica, Col. Villa Esmeralda, Tultitlán, C.P. 5491 Estado de México

ID $1^{\text {er }}$ Autor: Jorge, Rodríguez-Miramontes / ORC ID: 0000-0002-2879-7053, CVU CONACYT ID: 98187

ID $1^{\text {er }}$ Coautor: Maritza, Hernández-García / ORC ID: 0000-0003-0991-2869

DOI: $10.35429 /$ JPDL.2019.15.5.1.5

Recibido 20 de Marzo, 2019; Aceptado 30 Junio, 2019

\begin{abstract}
Resumen
El objetivo de esta investigación es analizar la vinculación entre las entidades gubernamentales y el sector industrial mediante las investigaciones (artículos publicados) generadas en conjunto con los investigadores que han pertenecido al Sistema Nacional de Investigadores (SNI) en el área VII (Ingeniería) al menos un año y están adscritos a una IES en México en sector público y privado. En este estudio se empleó una serie de datos procedentes del Science and Social Sciences Ciatation Index desarrollados por el Institute of Scientific Information (ISI)(ISI, 2012) hoy Thomson Reuters Web of Knowledge y corresponden a dos décadas de investigación científica mexicana. Con base en esta información se elaboró una base de datos de las publicaciones y citas de todos los artículos científicos que cuenten al menos un autor con dirección en México, publicados en el periodo de 1981 al 2011. Los resultados de esta investigación muestran que de los 26758 artículos publicados por los investigadores en el periodo de estudio solo el $8 \%$ de la investigación de las IES se genera en conjunto con entidades de gobierno mientras que la diminuta cantidad de $1.2 \%$ se genera en colaboración con el sector empresarial y un $80 \%$ se genera entre las mismas IES o con instituciones Internacionales.
\end{abstract}

Vinculación, Industria-academia, Ingeniería, SNI

\begin{abstract}
The goal of this research is to analyze the link between government entities and the industrial sector through research (published articles) generated jointly with researchers who have belonged to the National System of Researchers (SNI) in area VII (Engineering) at least one year and are attached to an HEI in Mexico in public and private sector. This study used a series of data from the Science and Social Sciences Ciatation Index developed by the Institute of Scientific Information (ISI) (ISI, 2012) today Thomson Reuters Web of Knowledge and correspond to two decades of Mexican scientific research. Based on this information, a database of publications and citations of all scientific articles that have at least one author with an address in Mexico, published in the period from 1981 to 2011, was prepared. The results of this research show that of the 26758 articles published by researchers during the study period, only $8 \%$ of HEI research is generated jointly with government entities while the tiny amount of $1.2 \%$ is generated in collaboration with the business sector and $80 \%$ is generated between the same HEIs or with international institutions
\end{abstract}

Linking, Industry-Academy, Engineering, SNI

Citación: RODRÍGUEZ-MIRAMONTES, Jorge \& HERNÁNDEZ-GARCÍA, Maritza. La investigación en las instituciones de educación superior: Vinculación con el sector gobierno e industria (1981-2011). Revista de Filosofía y Cotidianidad. 2019. 5-15: $1-5$

\footnotetext{
* Correspondencia del Autor (ingjorgerm@hotmail.com)

$\dagger$ Investigador contribuyendo como primer Autor.
} 


\section{Introducción}

La universidad a lo largo de la historia ha tenido grandes evoluciones, sin embargo, tiene tres funciones básicas determinadas las cuales son:

- $\quad$ Producción de conocimiento.

- $\quad$ Enseñanza del conocimiento acumulado.

- $\quad$ Preservación del conocimiento.

La producción del conocimiento, no podría generarse sin la investigación científica, ya que en las Instituciones de Educación Superior se debe motivar e incentivar a las nuevas generaciones para que con ello se genere un conocimiento progresivo de frontera y transmitirlo. La vinculación de la docencia con la investigación es un tema que ha generado un sinfín de trabajos, en uno de estos, Bernett (2008) señala que debe existir una transformación de la universidad, creando nuevas relaciones entre la investigación, saber y la docencia.

Sin embargo, la transformación de la universidad depende de muchas variables, por ejemplo, el nivel estructural de la institución, el presupuesto asignado y las políticas mismas de la institución. En este punto la política que está actualmente es la evaluación de los profesores. En este proceso, hay evidencia que muestra que el número de publicaciones del investigador tiene un considerable efecto en las decisiones de los evaluadores a la hora de determinar quiénes recibirán financiamiento (Arora \& Gambardella, 1998). A nivel micro, las universidades y centros de investigación emplean también el número de publicaciones y citas para supervisar el desempeño de los investigadores y para otorgar compensaciones, promociones y ascensos.

Sin embargo, el propósito de este articulo es dar a conocer la investigación y la docencia en las IES como un binomio que es capaz de generar vínculos con otras instituciones las cuales necesitan de los conocimientos creados para resolver problemas o crear tecnología para eficientizar sus procesos productivos. La vinculación entre universidades, centros de investigación, así como, con entidades gubernamentales y la industria, se debe entender como un proceso de perfeccionamiento del conocimiento existente y de la creación de nuevos conocimientos específicos y así aumentar la calidad de la docencia en las aulas.

\section{Metodología}

Para medir la vinculación con el Sector Gobierno e Industria, a través de la producción científica mediante la investigación, se considerarán los artículos generados en conjunto por los investigadores que han pertenecido al SNI en el área VII, al menos un año y están adscritos a una IES en México, en el sector público y privado asimismo con instituciones gubernamentales y empresas. Para generar la información necesaria se obtuvo acceso a información de 27667 investigadores de todos los campos del conocimiento que han sido parte del SNI, por lo menos un año de 1981 a 2011. Para medir los resultados de investigación, se obtuvo una base de datos de publicaciones y citas del Science and Social Sciences Citation Index, desarrollado por el Institute of Scientific Information (ISI) estas publicaciones fueron obtenidas mediante un cruce de información entre la base del SNI con la del ISI en el período mencionado obteniendo una muestra de 266451 artículos publicados en dicha ventana de tiempo.

\section{Discusión y resultados}

Para esta investigación se consideraron 19849 investigadores que publicaron un artículo al menos en el período de 1981 al 2011 mismos que pertenecen al área VII del SNI, en colaboración con 3706 organizaciones en las que se incluyen IES, entidades gubernamentales y empresas; las publicaciones totales de los investigadores en ese período fueron 25758 artículos de los cuales 306 fueron realizados en colaboración con la industria y 2077 artículos con entidades gubernamentales.

Como se puede observar en la figura 1. las publicaciones en estos dos sectores en la primera década de estudio (1981 a 1991) se mantuvieron constantes sin embargo a partir de los 90's la colaboración con las entidades gubernamentales en la investigación se incrementó considerablemente, pero para el trabajo en conjunto con la industria se puede apreciar que prácticamente se mantiene constante durante todo el período de estudio, en los tres últimos años con tan solo un pequeño crecimiento. 
Como se menciono anteriormente en la base de datos obtenida se tuvo registro de casi veinte mil investigadores mexicanos adscritos a una IES nacional, que la colaboración de otros investigadores de otras instituciones desarrolló las publicaciones aquí analizadas, es conveniente aclarar que para el objetivo de esta investigación no nos centraremos en los investigadores sino más bien en las instituciones a la cual están adscritos.

PUBLICACIONES EN COLABORACIÓN

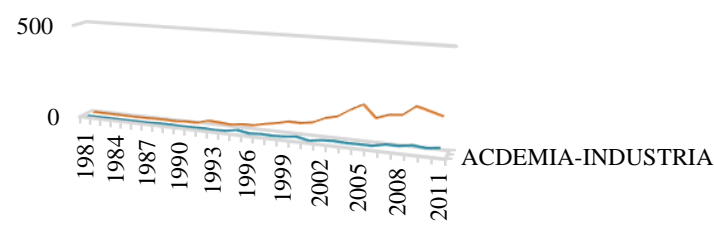

—ACDEMIA-INDUSTRIA $\square$ ACDEMIA-GOBIERNO

Figura 1 Publicaciones en colaboración con el sector gobierno e industrial con los investigadores en Ingeniería

En este sentido las siguientes tablas muestran la cantidad de artículos que fueron publicados por IES en colaboración con las entidades gubernamentales y del sector industrial. En la tabla 1 se muestran 81 instituciones las cuales por lo menos publicaron 2 artículos en el período de estudio, es conveniente aclarar que el número total de instituciones que publicaron fueron 239 significa que 158 instituciones publicaron un artículo en conjunto en un período de 30 años.

En la tabla 1 se organizó por el número de publicaciones de mayor a menor, en esta se aprecia que la Universidad Nacional Autónoma de México es la IES con mayor número de publicaciones 70, seguida por la Universidad Autónoma de Nuevo León con 55, el Centro de Investigación y de Estudios Avanzados (CINVESTAV) 41, después se encuentra el Instituto Politécnico Nacional con 35 artículos, es importante resaltar que la participación de IES privada en este tipo de vinculación se hace presente con el Instituto Tecnológico y de Estudios Superiores de Monterrey (ITESM) con 19 artículos.
Por parte del sector empresarial se puede observar que son pocas las industria que tienen más de 10 artículos en colaboración con la IES, las tres principales son Corporación Nemark S.A de S.V. con 25 artículos, seguida por Hylsa SA de $\mathrm{CV}$ con una publicación menos que la anterior, Intel Corporación es la tercera con 10 artículos al igual con Industrias Negromex, S.A. de C.V. después de estas empresas existen casos como COMEX, IPSAT, K \& E Technologies, S.A. de C.V. o Motorola que han publicado en conjunto con menos de 10 artículos.

Para la vinculación con el sector gubernamental, como ya se había mencionado la cantidad de artículos publicados es mayor en comparación con la industria, sin embargo, el comportamiento de las IES casi no vario mucho, como se observa en la Tabla 2. la UNAM es la IES con más vinculación con el sector gobierno con 786 publicaciones, seguida por la Universidad Autónoma Metropolitana UAM con 490 artículos, en tercer lugar el IPN con 345 seguido por el CINVESTAV con 134; las IES que figuran en este tipo de vinculación y no aparecieron en el anterior son: la Universidad Autónoma del Estado de Morelos con 109 participaciones, la Universidad Autónoma de San Luis Potosí con 78 publicaciones seguido por el Instituto Tecnológico de Toluca con 68.

Por parte de las Entidades Gubernamentales, el Instituto Mexicano del Petróleo (IMP) es la institución con más colaboraciones con las IES registrando un total de 1005 publicaciones, seguido por el Instituto Nacional de Investigaciones Nucleares (ININ) con 321 artículos, la Secretaria de Salud (SSA) con 115 colaboraciones, el Instituto Potosino de Investigación Científica y Tecnológica A.C. (IPICYT) con 111, el Instituto Mexicano de Tecnología del Agua (IMTA) con 73 publicaciones, el Instituto Mexicano del Seguro Social (IMSS) con 46 tantos y Petróleos Mexicanos (PEMEX) con 41 publicaciones.

Otro punto importante en la investigación es indagar que temas científicos desarrollan conjuntamente las IES con ambos sectores. 
La figura 2 muestra los trece tópicos de la ciencia con mayor publicación en el sector industrial, como se puede observar el tema principal de la investigación en conjunto es la Ciencia de Materiales con 95 publicaciones relacionadas con este tema, Metalurgia \& Ingeniería Metalúrgica con 50, Química con 40, Física con 37, Ciencias de la Computación con 30 y, Ingeniería Eléctrica-Electrónica con 23 artículos en este tema.

En la figura 3 se muestran las áreas de conocimiento en las cuales se centraron las publicaciones en colaboración con el Sector Gobierno como se puede apreciar química es el área de conocimiento en la cual se centra la colaboración con el gobierno con 834 artículos que pertenecen a esta área, Ciencia de los Materiales con 507, Física con 503, Ingeniería Química con 317, Energía y Combustibles con 171 publicaciones en esta área del

PUBLICACIONES POR ÁREA DE CONOCIMIENTO

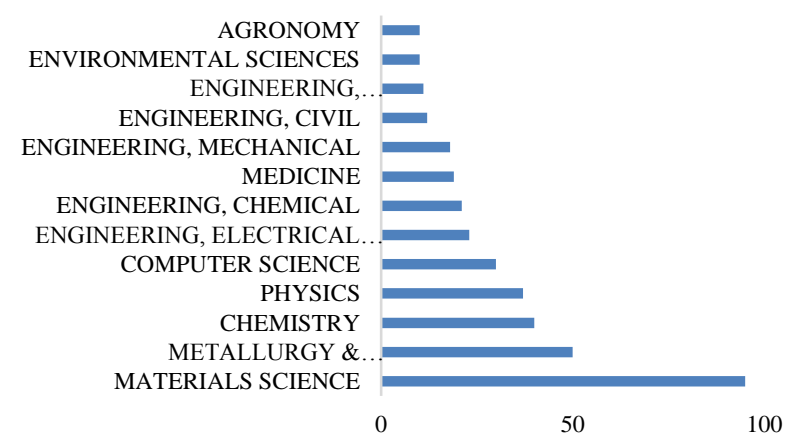

Figura 2 Número de publicaciones por área del conocimiento en ingeniería en colaboración con la industria

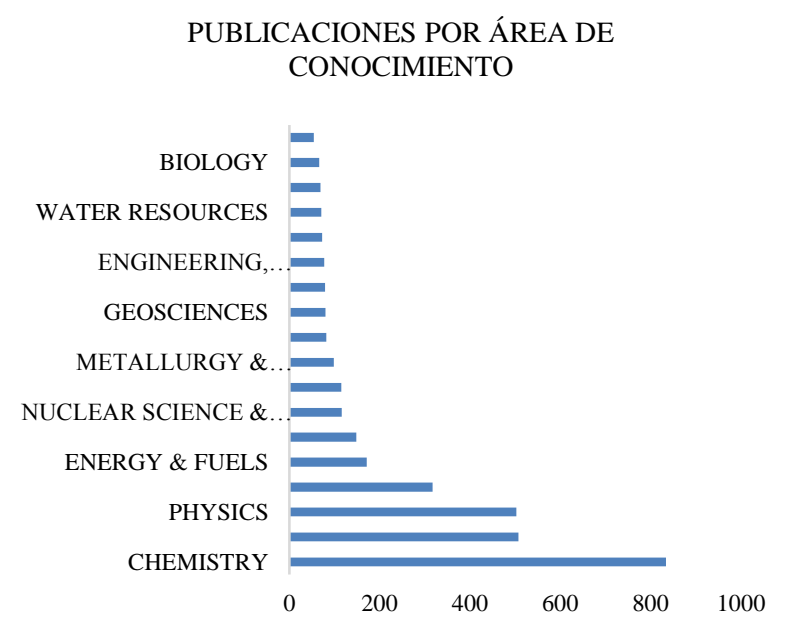

Figura 3 Numero de publicaciones por área del conocimiento en ingeniería en colaboración con el gobierno

\section{Conclusiones}

El objetivo de este trabajo es analizar la investigación como una vinculación de las actividades de las instituciones de educación superior de nuestro país con el sector industrial y gubernamental como resultado principal se encontró que las publicaciones en estos sectores en conjunto ocupan el $9.3 \%$ de la producción total, el $1.2 \%$ para la industria y el $8.1 \%$ con el gobierno esto significa que la actividad de vincularse con estos sectores es casi nula o que no es tan importante como otro tipo de colaboración, por ejemplo, la internacional o entre instituciones.

Sin embargo, lo importante de este trabajo es resaltar que existe esa vinculación con estos dos sectores para el desarrollo científico y tecnológico de México, aunque sea casi nula se puede aprovechar los casos de éxito para poder elevar los índices de vinculación con estos.

En este sentido es claro ver que en México los tópicos de investigación se centran en la Ciencia de los Materiales y Metalurgia y esto se refleja con el tipo de industria que se ha vinculado con el sector académico, la empresa Corporación Nemark S.A de S.V. se especializa en la manufactura y producción de componentes de aluminio para la industria automotriz, por otra parte la empresa Hylsa ahora Ternium Hylsa se dedica desde la extracción de mineral de hierro en sus propias minas y la fabricación de acero; Geográficamente estas dos empresas radican en el norte del país es claro ver que el éxito de la vinculación de la UANL y el CINVESTAV debido a la cercanía de sus campus han podido desarrollar investigación conjunta.

En el caso de la electrónica se tienen dos empresas que se ha conseguido una fuerte vinculación con la investigación, son el caso de Intel corporation y Motorola, para el caso de Intel, el Centro de Diseño en Guadalajara ha jugado un papel crítico en este tipo de vinculación, cosa similar ocurre a Motorola el diseño electrónico y la manufactura de componentes electrónicos son de gran importancia, en este sentido CINVESTAVGuadalajara ha tenido éxito en la colaboración con estas dos industrias. 
Por parte del sector gubernamental las áreas de ingeniería química y energía combustibles, química y física, claramente se puede explicar que el vínculo con el IMP institución relacionada con la investigación de los petroquímicos, genera la mayor cantidad de publicaciones en esos temas; también se encuentra el ININ y el IIE, sin embargo, es muy importante notar que existe la colaboración con el sector salud, ya sea con el IMSS o con los organismos de la secretaría de salud se ha generado un vínculo con la investigación.

Lo importante de esta investigación es dar a conocer los esfuerzos realizados por las IES para generar los vínculos de investigación con entidades gubernamentales y empresariales como es de esperarse las grandes universidades son quienes, han desarrollado más este tipo de vinculación, sin embargo es importante ver que pequeñas instituciones en comparación con las anteriores como los Institutos Tecnológicos (Morelia, Laguna o Celaya) o universidades de reciente creación como las politécnicas estén empezando a construir lazos fuertes de vinculación en la investigación con industria y el sector gobierno.

Por último, es importante resaltar los beneficios de este tipo de vinculación siempre serán en favor de nuestro país y en los estudiantes de ingeniería, ya que cuando se genera investigación con este tipo de entidades la transmisión del conocimiento es directa a la formación de nuestros futuros ingenieros, quienes tendrán la enorme responsabilidad de contribuir con el desarrollo tecnológico de nuestro país.

\section{Referencias}

Arora, A., \& Gambardella, A. (1998). Impact of NSF support for basic research in economics. EconWPA.

Bernett R. (2008). Para una transformación de la universidad nuevas relaciones entre investigación, saber y docencia. Octaedro Editorial, España..

\section{Anexo 1}

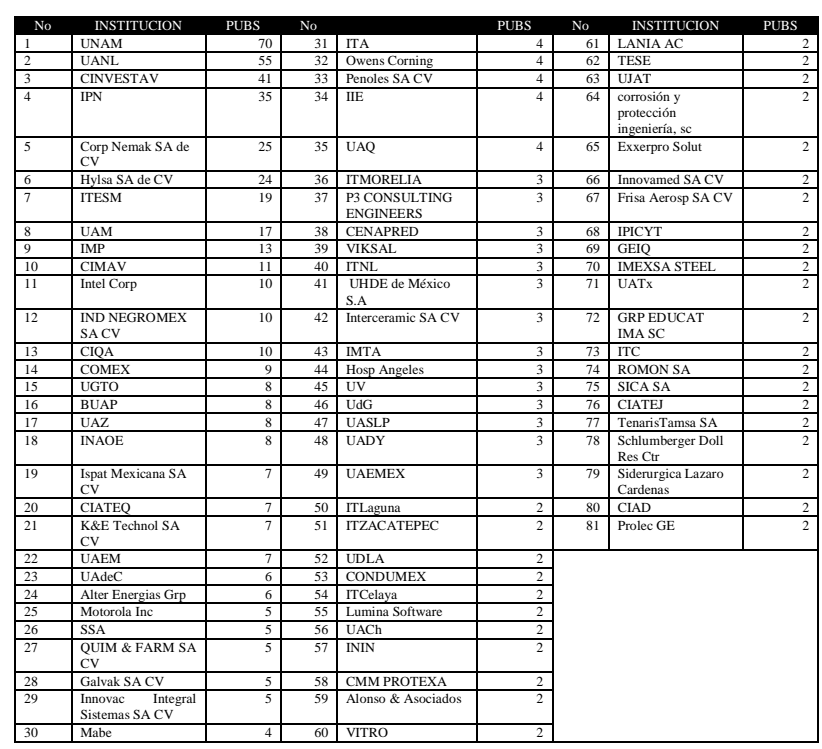

Tabla 1 Artículos publicados por institución en colaboración con la industria

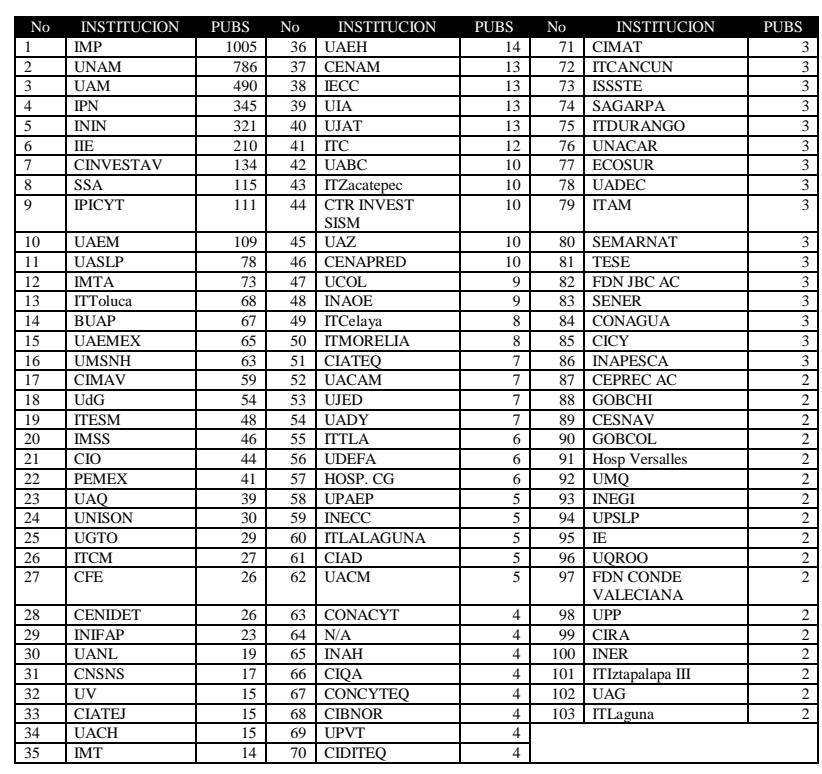

Tabla 2 Artículos publicados por institución en colaboración con sector gobierno 